\title{
Collaborative Filtering Recommendation Algorithm Based on User Evaluation and Potential Semantic Analysis
}

\author{
Jinlong Chen ${ }^{1+}$, Tao Zhang ${ }^{2}$ \\ ${ }^{1}$ Guangxi Key Laboratory of Cryptography and Information Security, Guilin University of Electronic \\ Technology, China \\ ${ }^{2}$ Guangxi Key Laboratory of Trusted Software, Guilin University of Electronic Technology, China
}

\begin{abstract}
: in order to solve the problem of unitary similarity and inexact data in traditional collaborative filtering algorithm, a collaborative filtering recommendation algorithm combining user evaluation and potential semantic analysis(CFRAEPSA) was proposed .The algorithm consists of two key parts. First, the TF-IDF idea is added into the weight calculation of labels, and the exponential decay function and time window are used to capture the changes of users' interests .Secondly ,it combines the potential semantic analysis algorithm to ensure the relevance of the recommended products.The experimental results show that the proposed algorithm is better than the traditional recommendation method in every evaluation index, and can ensure that the proposed algorithm can effectively improve the recommendation accuracy.
\end{abstract}

Keywords: Collaborative Filtering, TF - IDF, Exponential Attenuation Function, Latent Semantic Analysis, Item Similarity

\section{Introduction}

In recent years, the Internet presents a trend of rapid development, and users are exposed to more and more information. How to find out what users are interested in from mass data and recommend it to users has become the key .For users, can save search time, improve efficiency ;For enterprises, it can improve user satisfaction and attract more users .Therefore, the appropriate recommendation algorithm is particularly important .The most widely used is the recommendation algorithm based on content[1], collaborative filtering recommendation algorithm and hybrid recommendation algorithms[2], Among, the collaborative filtering algorithm based on user mainly calculating the similarity between the user and user behavior rating according to the user's personal history related products, finally find the nearest neighbor, predict the rating of the product, and then suggest the top $\mathrm{n}$ the highest rating to the user[3].However , there are many problems in the application of collaborative filtering recommendation algorithm. The user data that needs to be processed is often faced with sparsity, project cold start, lack of scalability [4] and other shortcomings, among which the inaccurate calculation of user similarity is also one of the main factors affecting the recommendation accuracy [5].In view of this problem, many scholars have carried out research . Considering that the difference in scores is an effective method to improve similar skew based item-based collaborative filtering module incremental update [6], it is recommended to dynamically adjust the incremental update list for similar items, and relevance is recommended to ensure the combination of potential semantic analysis algorithms .Method based on latent semantic analysis of user's attribute of the attribute information and project information match [7], latent

\footnotetext{
Corresponding author. Tel.: + 18069774991;

E-mail address: 18069774991@163.com
} 
semantic analysis of recommendation system using a variety of machine learning methods, including the matching method based on tag [8] and calculation method in the language and the main problem point analysis $[9,10]$ although the above method can reflect the user's interests and preferences in some ways, using the tag to solve the problems such as data sparse, cold start, without considering the user's interest will change over time, ignore the weight in the label in the project .In order to solve the above problems, this paper proposes a user-based collaborative filtering recommendation algorithm based on interest and potential semantic analysis .Firstly, the idea of TF-IDF is to calculate the user's weight item label, and the time window is integrated into the exponential decay function to reflect the user's interest changes more accurately . A potential semantic analysis algorithm based on keyword frequency will identify and analyze users with the same keyword attributes .Finally, consider the user's interests and keywords to evaluate the user's ultimate similarity, find out the target user's neighbors, predict project scores, and generate a list of recommendations.

\section{Related work}

\subsection{Traditional Collaborative Filtering Algorithm}

Traditional collaborative filtering algorithms based on user interests and preferences recommend personalized recommendation items based on the interests and preferences of target users .Secondly, by collecting the scores of the users' neighbors on the project, the possible scores of the current users on the project are predicted.Through iteration, predict the likely score value of the current user on the unrated item in the dataset .The scores will be predicted, sorted according to the scores, and the highest $\mathrm{N}$ items will be recommended to the current users.

In the process of similarity calculation, user-project score matrix $\mathrm{R}$ is used to represent the user's score information for the project, as shown in table 1.In it, the set of users is $\left\{u_{1}, u_{2}, \cdots, u_{m}\right\}$, the collection of items is $\left\{i_{1}, i_{2}, \cdots, i_{n}\right\}$, the value of the item not rated by the user is 0 .

Table 1: user-project scoring matrix

\begin{tabular}{ccccccc}
\hline & \multicolumn{7}{c}{ Item } \\
\cline { 2 - 7 } User & $\mathrm{v}_{1}$ & $\mathrm{v}_{2}$ & $\mathrm{v}_{3}$ & $\ldots$ & $\mathrm{v}_{\mathrm{n}-1}$ & $\mathrm{v}_{\mathrm{n}}$ \\
\cline { 2 - 7 } & 3 & 3 & 2 & $\ldots$ & 4 & 5 \\
$\mathrm{u}_{1}$ & 2 & 1 & 0 & $\ldots$ & 0 & 1 \\
$\mathrm{u}_{2}$ & 3 & 0 & 4 & $\ldots$ & 0 & 1 \\
\hline
\end{tabular}

The similarity between users is usually calculated by cosine similarity algorithm, Revised cosine similarity and other methods.

1) Cosine similarity algorithm

$$
\operatorname{sim}(u, v)=\frac{\sum_{i \in T_{u, v}} r_{u, i} \cdot r_{v, i}}{\sqrt{\sum_{i \in T_{u, v}} r_{u, i}{ }^{2} \cdot \sum_{i \in T_{u, v}} r_{u, v}^{2}}}
$$

2) Revised cosine similarity

$$
\operatorname{sim}(u, v)=\frac{\sum_{i \in T_{u, v}}\left(r_{u, i}-\bar{r}_{u}\right)\left(r_{v, i}-\bar{r}_{v}\right)}{\sqrt{\sum_{i \in T_{u, v}}\left(r_{u, i}-\bar{r}_{u}\right)^{2}} \sqrt{\sum_{i \in T_{u, v}}\left(r_{v, i}-\bar{r}_{v}\right)^{2}}}
$$

In equations (1) to (2), represents the rating information of user $u$ on project $v$, represent the average score of user $\mathrm{u}$ and $\mathrm{v}$ respectively, and resent the item set of user $\mathrm{u}$ and $\mathrm{v}$ respectively, and represents the item set of user $u$ and $v$ jointly rated. 
Score prediction based on user similarity, as shown in equation (3);

$$
\operatorname{preds}(u, v)=\bar{r}_{u, v}+\frac{\sum_{u^{\prime} \in U_{\text {sim }}(u)} \operatorname{sim}\left(u, u^{\prime}\right) \cdot\left(r_{u^{\prime}, v}-\bar{r}_{u, v}\right)}{\sum_{u^{\prime} \in U_{\text {sim }}(u)} \operatorname{sim}\left(u, u^{\prime}\right)}
$$

Among them, $\bar{r}_{u, 1}$ for users to its has the average score of grading attractions ; $U_{s i m(u)}$ is the set of users whose similarity with user $u$ is greater than $0 ; u^{\prime}$ is one of its users ; $\operatorname{sim}\left(u, u^{\prime}\right)$ is the similarity between user $u_{\text {and user }} u^{\prime}$; $\operatorname{preds}(u, v)$ gives the prediction of the target user $u$ overall score of the target attraction $v$.

\subsection{Potential Semantic Analysis Algorithm Based on Keyword Frequency}

Potential semantic analysis techniques are represented by vector space algorithms. Suppose there are N comments, expressed as $D \stackrel{=}{=}\left\{d_{1}, d_{2}, \cdots, d_{n}\right\}$; M keywords, expressed as $S=\left\{s_{1}, s_{2}, \cdots, s_{m}\right\}$;Comment $d_{j}$ can be expressed as $d_{j}=\left\{w_{1, j}, w_{2, j}, \cdots, w_{m, j}\right\}$, where $w_{i, j}$ represent the weight of the ${ }^{i}$ keyword $S_{i}$ in the comment ${ }^{D_{j}}$.TF-IDF (Term Frequency-Inverse Document Frequency), a classical word frequency-inverse text frequency technique in the field of information retrieval, is used to calculate the weight of keywords. Given keywords $\mathrm{x}$ and comment $\mathrm{y}$, the calculation formula of keyword frequency is as follows:

$$
\begin{aligned}
F T(x, y) & =\frac{f(x, y)}{\max r e s t(x, y)} \\
\max r e s t(x, y) & =\max (f(z, y)) \\
z & \in w(x, y)
\end{aligned}
$$

Among them, $T F(x, y)$ is the frequency of qualified words of keywords $x$ in comment $y, f(x, y)$ is the exact number of times that keywords $x$ has appeared in comment $y, w(x, y)$ is the set of all kinds of keywords except $x$ in comment $y$, and maxrest $(x, y)$ is the maximum number of occurrences of keywords other than $x$ in comment $y$.

Intuitively, the weight of keywords in the title is different from that in the content, and the keywords in the title are relatively more important.

1) Keyword weight formula

$$
\begin{gathered}
\operatorname{IDF}(x)=\log \frac{n}{n(x)} \\
T F-I D F(x, y)=T F(x, y) \cdot \operatorname{IDF}(x)
\end{gathered}
$$

$\operatorname{IDF}(x)$ is the reverse frequency of weakening the keyword weight in multiple comments, and $n(x)$ is the number of comments containing the keyword $x$.

2) Keyword frequency calculation formula

$$
\begin{aligned}
T F^{\prime}(x, y) & =\frac{h \cdot f t(x, y)+f c(x, y)}{\max r e s t(x, y)} \\
T F-I D F(x, y) & =T F^{\prime}(x, y) \cdot I D F(x)
\end{aligned}
$$

Where, $f t(x, y)$ is the occurrence of the keyword $x$ in the title of comment $y, f c(x, y)$ is the occurrence of the keyword $x$ in the content of comment $y$, and the $h$ is the weight parameter.

At the heart of the potential semantic analysis is the dimensionality reduction process, which user the cosine formula to calculate the similarity between two reviews $d_{i}$ and $d_{j}$. 


$$
\operatorname{sim}\left(d_{i}, d_{j}\right)=\frac{\sum_{s=1}^{k} w_{s, i} \cdot w_{s, j}}{\sqrt{\sum_{s=1}^{k} w_{s, i}{ }^{2} \cdot \sqrt{\sum_{s=1}^{k} w_{s, j}{ }^{2}}}}
$$

$w_{s, i}$ represent the weight of the keyword $s_{i}$ in the comment $d_{i}$.

\section{Experimental Scheme Design}

\subsection{Experimental Data Set}

In this study, with the permission of the website, scenic spots of Sanya , Beijing , Hangzhou and Guilin were extracted from the travel service Websites Ctrip (https://www.ctrip.com) and(https://www.tuniu.com), and users' comments on these scenic spots were collected .The tourism data set of Sanya includes 118 scenic spots, 28,460 users, and 60,625 users' comments on scenic spots .Beijing tourism data set includes 248 scenic spots, 68,995 users, and 156,639 user rating records for scenic spots .The Guilin tourism data set includes 161 scenic spots, 11,478 users, and 25,779 user ratings of scenic spots .Hangzhou tourism data set includes 198 scenic spots, 56,639 users, and 87,990 user ratings of scenic spots.

An overview of the dataset statistics is shown in table 1.

\begin{tabular}{|c|c|c|}
\hline & User Number & Comment \\
\hline Websites Ctrip & 84622 & 230471 \\
\hline TuNiu & 57300 & 104788 \\
\hline
\end{tabular}

Fig.1: Datasets

\subsection{Evaluation Index}

To test the effectiveness of the proposed similarity calculation method based on multiple scores, the experimental results were evaluated using the four most common indicators: precision, recall rate, mean absolute error and $\mathrm{F}$ measure.

1) precision :

$$
p r e=1-\frac{\sqrt{\frac{\sum_{u_{i,}, s_{i} \in E}\left(r_{i j}-\hat{r}_{i j}\right)^{2}}{|E|}}}{4}
$$

2) recall rate :

$$
\text { recall }=\frac{\sum_{u \in E}|R(u) \cap S(u)|}{\sum_{u \in E}|S(u)|}
$$

3) mean absolute error :

$$
M A E=\frac{1}{|E|} \sum_{u_{i j}, s_{i j} \in E}\left|r_{i j}-\widehat{r}_{i j}\right|
$$

4) $\mathrm{F}$ measure :

$$
F(N)=\frac{2 \times \text { precision } \times \text { recall }}{\text { precision }+ \text { recall }}
$$


Where, E is the test set, $|E|$ is the number of elements in the set, $\left(u_{i}, s_{j}\right)$ represents a user-attraction pair, $r_{i, j}$ is the real rating of scenic spot $s_{i}$ by user $u_{j}, \hat{r}_{i, j}$ is user $u_{i}{ }^{\prime}$ prediction score of scenic spot $s_{j}$.The higher the accuracy and the smaller the mean absolute error, the smaller the gap between the predicted score and the real score, and the better the recommendation effect. In some cases, the indicators of accuracy and recall rate are not good enough to evaluate the performance of the recommendation system .A common solution is to combine accuracy with recall, where an $F(N)$ value is used to measure recommended performance.

\subsection{Experimental Scheme}

Every time a user enters a travel page, they need to make recommendations .The project-based collaborative filtering algorithm gives the recommended candidate set according to the score.The potential semantic analysis algorithm based on the number of keywords takes the user evaluation set as the element, calculates the similarity between the current user evaluation and the evaluation set, generates the final recommendation list, and updates the similarity list between the items. This paper USES three algorithms as comparison algorithms: User-Evaluated Collaborative Filtering Algorithm(UECFA), Item-based Collaborative Filtering Algorithm (ICFA) and Latent Semantic Analysis Algorithm(LSAA).

\section{Analysis of Experimental Results}

When keyword frequency is used to indicate the importance of keywords in a comment, the keyword weight in the title is different from that in the content .In this experiment, set different parameter values $h$ to increase the weight of keywords in the title, and use index $\mathrm{F}$ to measure the comprehensive performance of the system. The higher the value of $\mathrm{F}$, the better the recommended performance of the system.

As can be seen from figure 1 , when $0.8 \ll h \ll 1.2$, the recommendation performance improves as the weight of keywords in the title increases ; when $h \gg 1.2$, As the weight of keywords in the title continues to increase, the recommendation performance starts to decline. When $h=1.2$, the performance of the algorithm reaches the optimal value. Therefore, set the parameter value $h=1.2$ in this experiment.

$\mathrm{F}(\mathrm{N})$

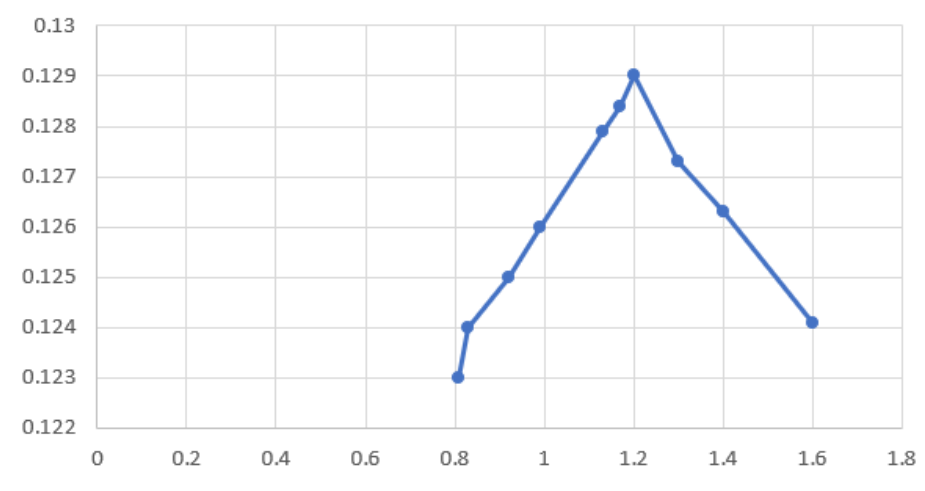

Fig.3: The influence of weighting factors

The experimental results are shown in figure 2. In this experiment, two indexes, precision, recall rate were compared among the four methods of CFRAEPSA,UECFA, ICFA, LSAA, respectively. It can be seen from the experiment that with the increase of the length of the recommendation list, the recommendation precision of the algorithm began to decrease .The experimental results show that the precision, recall rate values of CFRAEPSA algorithm are significantly higher than those of other algorithms under different recommendation quantities. 


\section{recall rate}
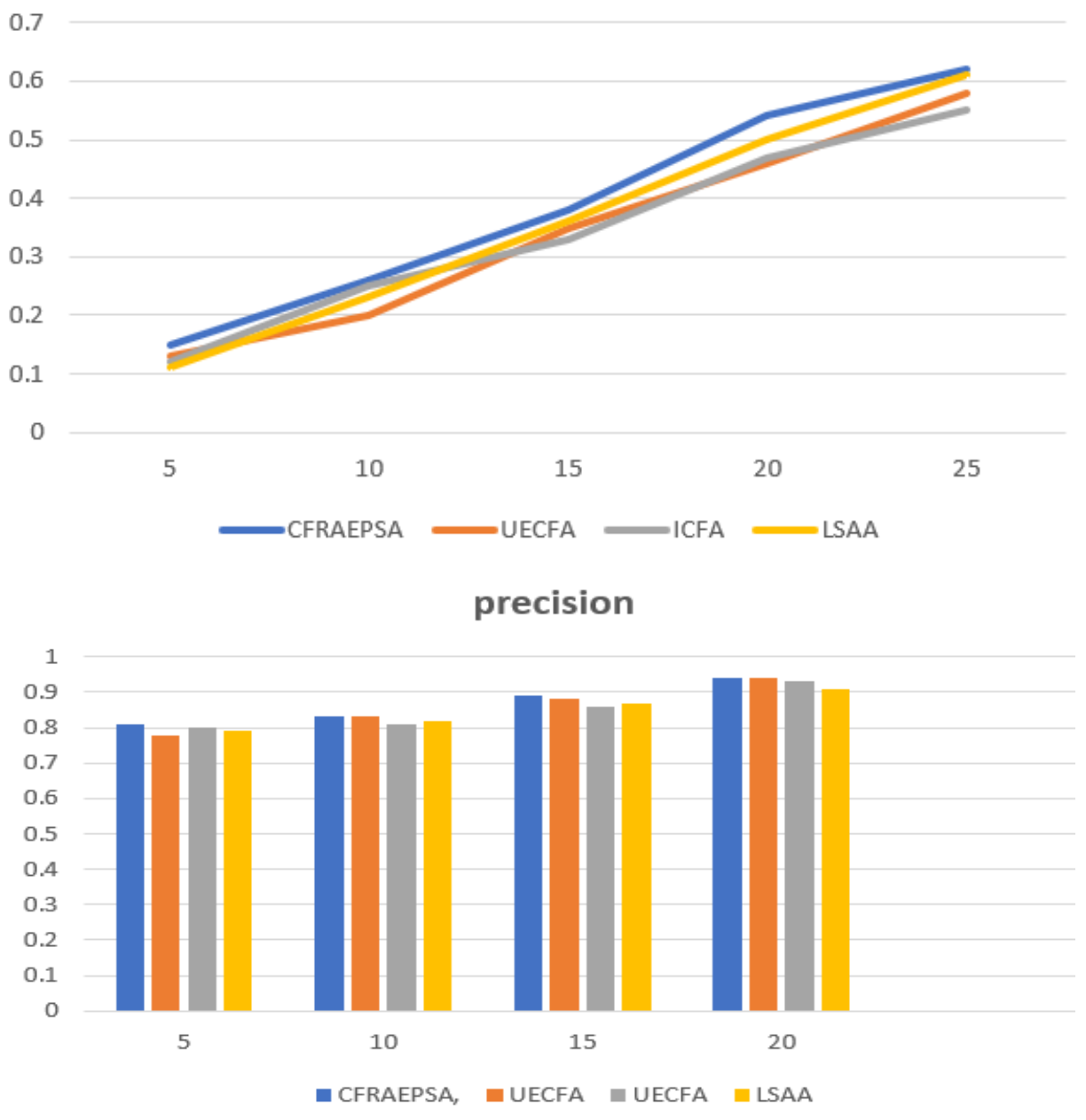

$\begin{array}{ccc} & \text { mean absolute error } & \text { F measure } \\ \text { CFRAEPSA } & 0.578 & 0.875 \\ \text { UECFA } & 0.567 & 0.873 \\ \text { ICFA } & 0.576 & 0.871 \\ \text { LSAA } & 0.584 & 0.873\end{array}$

Fig.4: Recall and precision of different algorithms

\section{Summary}

This study proposes a collaborative filtering recommendation algorithm based on user evaluation and potential semantic analysis to improve the calculation of user or user similarity .The user evaluation of scenic spots in Beijing, Hangzhou, Guilin and Sanya in China was collected from the Internet, and a comparative experimental scheme was designed, and the prediction effect was evaluated through the indicators of accuracy and recall experimental results show that compared with other recommendation algorithms, this algorithm makes the similarity calculation between users and users more accurate, thus making the score prediction more accurate .In the future, it can be considered to add real-time factors and other factors such as user interest and attraction labels to calculate the similarity between users or objects more accurately, so as to further improve the accuracy of collaborative filtering. 


\section{Acknowledgements}

This research work is supported by the grant of Guangxi science and technology development project (No: AB17195027), the grant of Guangxi scientific research and technology development plan project (No: AB18221011), the grant of Guangxi Key Laboratory of Trusted Software of Guilin University of Electronic Technology (No: KX201620), the grant of Guilin science and technology project Research and application demonstration of key technologies of somatosensory interaction based on gesture (No: 20180107-4).

\section{References}

[1] Yang kai et al., personalized recommendation of scientific literature based on content and collaborative filtering. Information technology, 2019.43 (12) : 11-14.

[2] Xu j, et al. Hybrid recommendation algorithm based on collaborative filtering and cryptic model. Computer technology and development, 2020. 30 (02) : 52-57.

[3] Lu hang, shi zhibin and liu zhongbao, a collaborative filtering recommendation algorithm that integrates user interest and score differences. Computer engineering and application, 2020.56 (07) : 24-29.

[4] Qiao yu, research on recommendation algorithm for cold start problem, 2018, nanjing university of posts and telecommunications, pp. 63.

[5] Zhao wentao and lv xia. Collaborative filtering algorithm based on user characteristics and similar confidence. Measurement and control technology, 2019.38 (08) : 95-98 + 102.

[6] Xu ji et al., hybrid recommendation algorithm based on collaborative filtering and crypto-semantic model. Computer technology and development, 2020(02): 1-8.

[7] Xu qidong, research on personalized recommendation system based on cryptic meaning model, 2019, guangdong university of technology, pp. 74.

[8] Dong chenxi, research on automatic summary method of short texts based on deep learning, Beijing university of posts and telecommunications, 2019, p. 66.

[9] Liu hui, wan chengfeng and wu xiaohao, a hybrid recommendation algorithm based on incremental collaborative filtering and potential semantic analysis. Computer engineering and science, 2019. 41(11): 2033-2039.

[10] Wang licai, meng xiangwu, zhang yujie. Context-aware recommendation system [j]. Software journal, 2012. 23 (1) : 1-20. 